\title{
Simulação do monitoramento de veículos em uma rede LoRaWAN usando NS-3
}

\author{
João P. Pastório ${ }^{1}$, Álvaro R. Castro e Souza ${ }^{1}$, Edson T. de Camargo ${ }^{1}$ \\ ${ }^{1}$ Colegiado de Engenharia de Computação \\ Universidade Tecnológica Federal do Paraná (UTFPR) - Toledo, PR - Brasil \\ joaopastorio@alunos.utfpr.edu.br, alvarosouza@utfpr.edu.br, edson@utfpr.edu.br
}

\begin{abstract}
Resumo. Com o aumento no número de aplicações voltadas à Internet das Coisas sendo desenvolvidas, surge a necessidade de se projetar uma infraestrutura de rede adequada para suportar as suas exigências. Com isso, esse estudo avalia em ambiente simulado o desempenho de uma rede LoRaWAN submetida a diferentes cenários de operação no contexto de objetos em movimento. São realizadas alterações no nível de interferência presente no meio de propagação do sinal e variações no número de pontos de acesso que a infraestrutura compreende. São abordados desde cenários com baixa interferência e poucos pontos de acesso até cenários com várias obstruções e muitos pontos de acesso. A simulação ocorreu através do simulador de código aberto NS-3 e seus módulos para representar cenários com diferentes níveis de interferência e dispositivos se comunicando com números variados de pontos de acesso. Os resultados obtidos apresentam o comportamento dos dispositivos presentes na rede durante a comunicação incluindo a porcentagem de pacotes perdidos em cada um dos cenários avaliados.
\end{abstract}

\section{Introdução}

O termo Internet das Coisas ou Internet of Things (IoT) está associado a dispositivos que apresentam a capacidade de trocar informações entre si ou com pontos de acesso. Esses dispositivos muitas vezes estão relacionados a diversas aplicações do nosso cotidiano, como por exemplo lâmpadas inteligentes, sensores de presença e fumaça, ou a aplicações urbanas, como no monitoramento de veículos da coleta seletiva que está em desenvolvimento na UTFPR, campus Toledo, como fruto de uma parceria com o município [de Camargo et al. 2021a, da Silva et al. 2020]. Nesse âmbito, as tecnologias associadas às redes de baixo consumo energético e ampla área de abrangência (Low Power Wide Area Network, LPWAN) estão sendo muito empregadas. A tecnologia LoRa, derivação para Long-Range, é uma das tecnologia de implementação LPWAN. Essa, por sua vez, utiliza o protocolo de rede LoRaWAN para o gerenciamento da comunicação entre dispositivo IoT e pontos de acesso, também chamados de gateways. A rede LoRaWAN permite a implantação de uma rede de baixo custo, flexível e em grande escala por utilizar uma largura de banda não licenciada [Rossato et al. 2020].

Um dos quesitos chave em uma rede IoT está na sua capacidade de suportar um grande número de dispositivos em operação. Ao se implementar uma rede de sensores, como a LoRaWAN, busca-se a criação de uma infraestrutura robusta e com uma menor suscetibilidade a erros [Mekki et al. 2019]. Então, com a utilização de simulações, é possível projetar diversos cenários de operação dos dispositivos nessa rede, analisando 
futuros problemas que podem ser encontrados na prática e assim maneiras de evitá-los. Uma vez que são encontrados os cenários adequados para a projeção da uma infraestrutura de rede, a sua implementação pode ser realizada com um menor custo e podendo atingir um maior desempenho. Por fim, conhecer os limites de uma rede IoT antes do seu desenvolvimento pode contribuir com futuras aplicações que vão operar em sua infraestrutura e contribuir para desenvolver soluções confiáveis [Pastório et al. 2020].

Existem algumas pesquisas relacionadas a análise em simulação de uma rede LoRaWAN. Em [Ortiz et al. 2019] compara-se o desempenho simulado e prático de um dispositivo final se comunicando com um ponto de acesso, enquanto em [Abeele et al. 2017] realiza-se a detecção de erros provenientes de um grande número de dispositivos simultaneamente conectados e enviado pacotes de dados ao ponto de acesso. Apesar das abordagens realizadas por essas pesquisas, outros fatores impactam no desempenho de uma rede LoRaWAN e na perda de pacotes enviados pelos dispositivos. Um desses fatores são as perdas causadas pela distância entre dispositivo e ponto de acesso, influenciadas pelo ambiente que o sinal se propaga [Goldsmith 2005]. Essa, por sua vez, apresenta relação com fenômenos de sombreamento ou desvanecimento do sinal.

Este trabalho busca investigar o impacto de diferentes ambientes e cenários de propagação no desempenho da rede LoRaWAN. Em particular, deseja-se estudar tal impacto em uma aplicação onde há objetos móveis, tais como os caminhões da coletiva seletiva. O trabalho faz uso de módulo LoRaWAN desenvolvido por Magrin [Magrin 2016] para o simulador de redes de código aberto baseado em eventos discretos Network Simulator 3 (NS-3). O desempenho e operação de uma rede LoRaWAN submetida a cenários e condições diversas é analisado de forma simulada. Examina-se também o impacto da alteração no meio em que o sinal se propaga, com o objetivo de obstruir o campo de visão entre dispositivo e ponto de acesso, e simular os efeitos de sombreamento e desvanecimento de sinal.

\section{Método}

\subsection{Tecnologia LoRa e LoRaWAN}

A tecnologia LoRa consiste em uma modulação que opera na camada física das redes com amplo alcance e baixo consumo de potência LPWAN. Essa rede se caracteriza pela sua extensa área de abrangência, estando diretamente relacionada aos níveis de obstruções presentes no meio, podendo chegar a quilômetros de distância. Essas redes também apresentam um baixo consumo energético, o que é possível pelo envio de pequenas quantidades de dados em um grande intervalo de tempo, o que reduz o tempo de ativação dos dispositivos e aumenta a vida útil de suas baterias [Mekki et al. 2019].

O protocolo LoRaWAN foi criado para gerenciar a comunicação dos dispositivos que utilizam a tecnologia LoRa em sua camada física. Nesse protocolo são definidas algumas especificações, como a classe dos dispositivos e como os mesmos enviam suas mensagens [LoRa Alliance 2017]. Outro aspecto definido é a arquitetura da rede LoRaWAN, que se baseia na topologia estrela, apresentada na Figura 1. Uma rede LoRaWAN apresenta dispositivos finais, como sensores e atuadores, que se comunicam com pontos de acesso, conhecidos como gateways, que estão diretamente conectados aos servidores responsáveis por gerenciar a distribuição dos dados para as aplicações finais. 


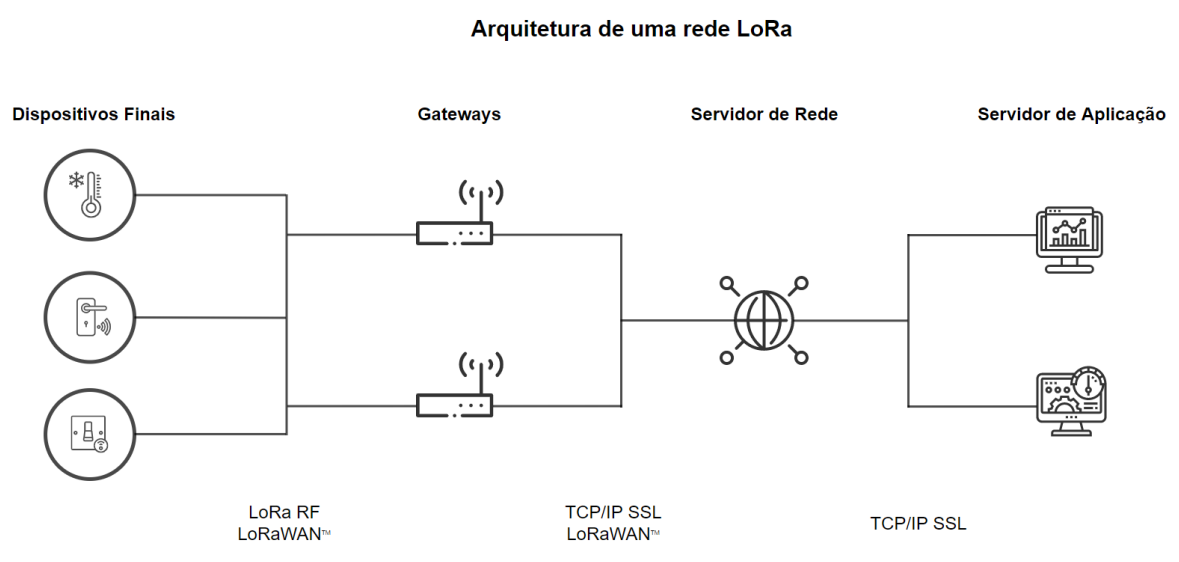

Figure 1. Arquitetura de uma rede LoRaWAN

\subsection{NS-3 e Módulo LoRaWAN}

O NS-3 é formado por diferentes módulos responsáveis por simular cada aspecto de uma rede de dispositivos. O módulo LoRaWAN elaborado por [Magrin 2016] fornece uma série de classes para reproduzir cada componente de uma rede LoRaWAN. O modelo de perda de caminho por distância logarítmica é utilizado para analisar o impacto da distância na potência do sinal transmitido [Goldsmith 2009], seguindo a formulação matemática apresentada na equação 1 .

$$
L=L_{0}+10 n \log _{10}\left(\frac{d}{d_{0}}\right)
$$

A simulação de obstruções no meio é realizada pela alteração no expoente de perda de caminho $n$ da equação 1, assim como pela utilização do modelo TR 45.820, do sombreamento presente no módulo LoRaWAN [Magrin 2016] e modelos para representação de construções do próprio NS-3. A partir dessas considerações, são definidos os seguintes cenários de operação:

- Cenário 0: $n=3,5$ sem construções ou sombreamento, simulando um ambiente aberto;

- Cenário 1: $n=3,75$ com poucas construções e sombreamento, simulando um ambiente pouco urbanizado;

- Cenário 2: $n=4$ com muitas construções e sombreamento, simulando um ambiente urbano.

\section{Resultados}

O primeiro resultado foi avaliado para os 3 cenários mencionados considerando um dispositivo final em movimento e alteração no número de gateways, como mostrado na Figura 2. Obtém-se resultados quanto a taxa de pacotes transmitidos e recebidos durante a comunicação do dispositivo final em cada ponto do deslocamento. Primeiramente, para o cenário 0 com apenas o Gateway 1 em operação, depois sendo considerado os Gateways 1 e 2 e assim sucessivamente, até ser realizada para os 3 cenários e os 5 gateways. $\mathrm{O}$ posicionamento dos gateways foi escolhido de acordo com projetos em desenvolvimento 
entre a Universidade Tecnológica Federal do Paraná e a prefeitura de Toledo Paraná para implantação de uma rede LoRaWAN na cidade [de Camargo et al. 2021b].

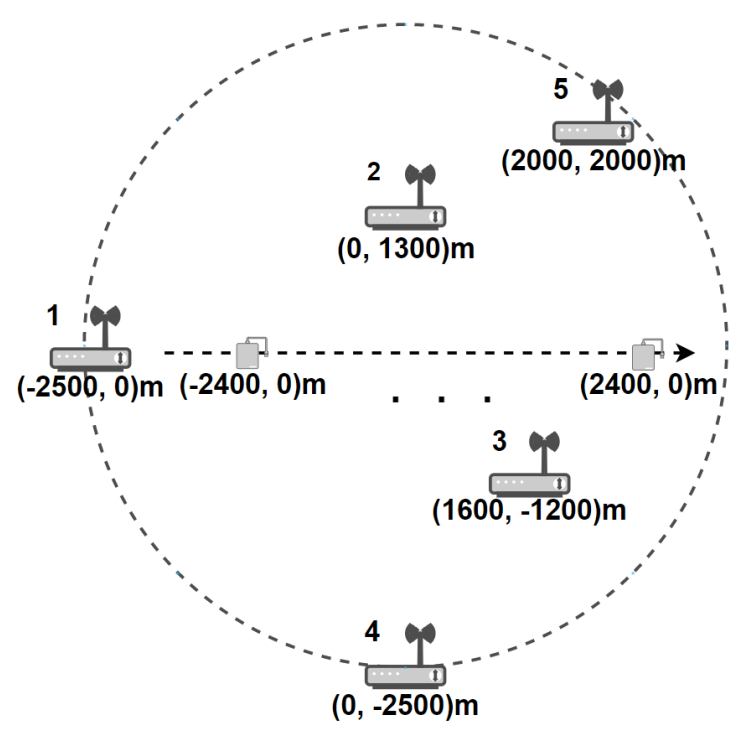

Figure 2. Representação do cenário simulado

Para o cenário 0, conforme o dispositivo final começa a se distanciar do Gateway 1 a porcentagem de pacotes recebidos começa a reduzir, quando considerado apenas um gateway essa porcentagem que começa em $100 \%$ termina em aproximadamente $30 \%$, apresentando uma queda de quase $70 \%$ de pacotes recebidos. Conforme o número de gateways é aumentado para o mesmo cenário, nota-se que a porcentagem de pacotes recebidos também aumenta, ficando entre $90-100 \%$ para 3, 4 e 5 gateways. Isso ocorre por conta do ambiente não apresentar obstruções, sendo limitados apenas pela distância percorrida pelo sinal.

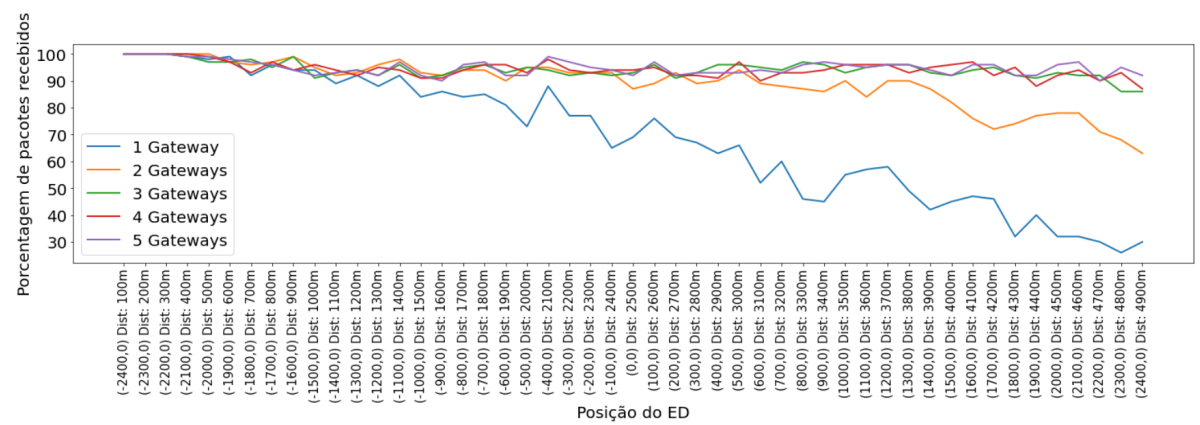

Figure 3. Simulação para o cenário de operação 0

Já no cenário de operação 1, considerando algumas poucas obstruções e apenas com o Gateway 1 em operação, no final do trajeto a porcentagem de pacotes recebidos é reduzida para aproximadamente $0 \%$. Ao considerar 3, 4 e 5 gateways essa porcentagem fica por volta de $80 \%$. Diferente do cenário 0, agora que estão presentes obstruções alguns pacotes de dados sofrem interferência e não são recebidos.

No cenário 2, considerando um ambiente urbano, com alto índice de obstruções, sombreamento e construções, a porcentagem de pacotes recebidos permanece por volta 


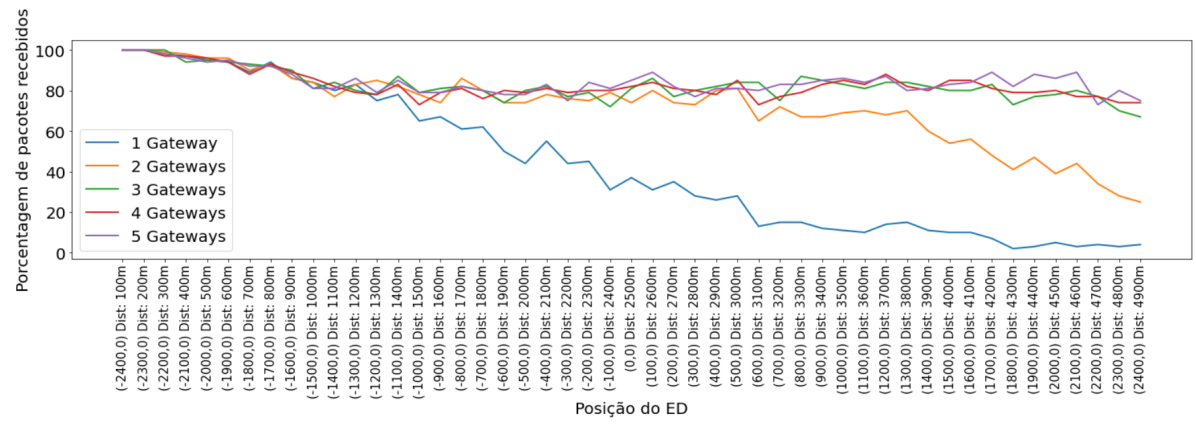

Figure 4. Simulação para o cenário de operação 1

de 50\% para 3, 4 e 5 gateways. Com apenas o Gateway 1 em operação a porcentagem chega a $0 \%$ de pacotes recebidos em uma distância muito menor do que para o cenário 1 analisado.

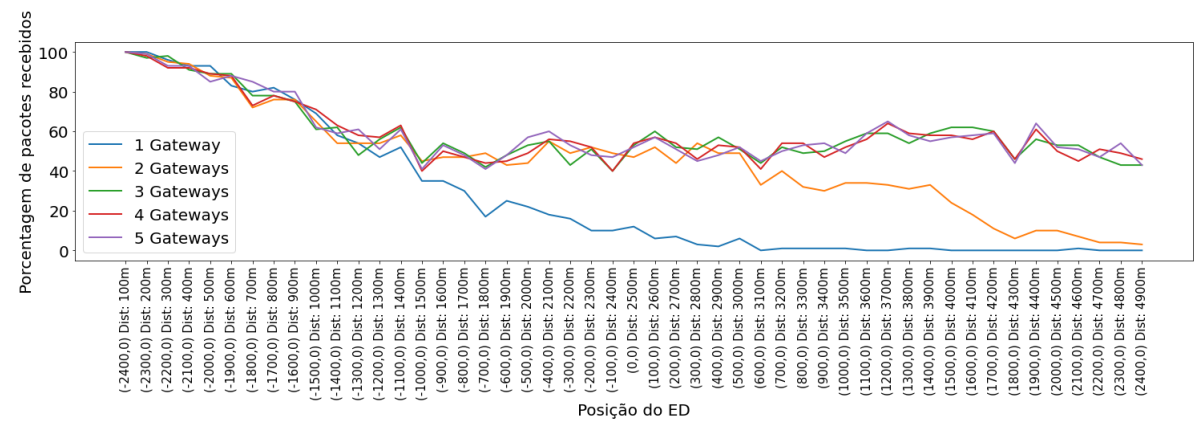

Figure 5. Simulação para o cenário de operação 2

Os resultados revelam que em ambientes com poucas obstruções o aumento no número de gateways se mostra suficiente para contornar a redução na porcentagem de pacotes recebidos. Porém, em ambientes com um alto índice de interferência pode ser necessário realizar alguma outra abordagem além da utilização de mais gateways para manter o desempenho da rede. Identificando uma redução de aproximadamente $50 \%$ na porcentagem de pacotes recebidos quando se tem 5 gateways em operação, indo de 90$100 \%$ no cenário 0 , considerando um ambiente com poucas obstruções, para 40-50\% no cenário 2 ao considerar várias construções e sombreamento do no meio que o sinal se propaga.

\section{Conclusões}

Com esse estudo foi possível verificar, em ambiente simulado, o comportamento de uma rede LoRaWAN submetida a diferentes cenários de operação para o monitoramento de veículos em movimento. A partir das simulações realizadas percebe-se que o aumento no número de gateways presentes na rede impacta diretamente na porcentagem de pacotes recebidos quando lida-se com um objeto em diferentes posições. Porém, o cenário de operação que os dispositivos e ponto de acesso estão presentes pode limitar a porcentagem de pacotes recebidos pelas diferentes interferências que passam a agir sobre o sinal. Esse problema pode ser reduzido com a utilização de algoritmos de retransmissão. Em [Almeida et al. 2021] é apresentada a expansão dessas simulações, considerando um al- 
goritmo de retransmissão para verificar seu impacto na porcentagem de pacotes recebidos, compilando as análises simuladas aqui expostas com uma análise prática.

\section{Agradecimentos}

Os autores agradecem a UTFPR e a Prefeitura de Toledo ao apoio financeiro pelo edital N. ${ }^{\circ}$ 05/2020 PROBIC-TD e o Acordo de Cooperação 001/2020, respectivamente.

\section{References}

Abeele, F. V. d., Haxhibeqiri, J., Moerman, I., and Hoebeke, J. (2017). Scalability analysis of large-scale lorawan networks in ns-3. IEEE Internet of Things Journal, PP.

Almeida, T. V. O., Pastório, J. P., Álvaro R. C. Souza, Spanhol, F. A., and de Camargo, E. T. (2021). Em busca de um algoritmo de retransmissão em redeslorawan. V Courb.

da Silva, M., de Souza, J., Almeida, T., Álvaro Souza, Spanhol, F., and Camargo, E. (2020). Avaliação de dispositivos de rastreamento em uma rede lorawan no contexto de cidades inteligentes. In Anais do IV Courb, pages 1-14, Porto Alegre, RS, Brasil. SBC.

de Camargo, E. T., Spanhol, F. A., and e Souza, Á. R. C. (2021a). Deployment of a lorawan network and evaluation of tracking devices in the context of smart cities. $J$. Internet Serv. Appl., 12(1):8.

de Camargo, E. T., Spanhol, F. A., and e Souza, Á. R. C. (2021b). Deployment of a lorawan network and evaluation of tracking devices in the context of smart cities. $J$. Internet Serv. Appl., 12(1):8.

Goldsmith, A. (2005). Wireless Communications. Cambridge University Press, New York.

Goldsmith, T. S. (2009). Comunicações sem fio, Princípios e Práticas. Person Prentice Hall, São Paulo.

LoRa Alliance (2017). LoRaWAN 1.1 specification. LoRa Alliance, Beaverton, Oregon, Estados Unidos.

Magrin, D. (2016). Network level performances of a lora system. Master's thesis, Università degli Studi di Padova, Pádua. Orientador: Lorenzo Vangelista.

Mekki, K., Bajic, E., Chaxel, F., and Meyer, F. (2019). A comparative study of lpwan technologies for large-scale iot deployment. 5:1-7.

Ortiz, F. M., Almeida, T., Ferreira, A. E., and Costa, L. H. (2019). Caracterização de desempenho de uma rede lora em ambientes urbanos: Simulação vs. prática. In Anais do III Courb, pages 167-180, Porto Alegre, RS, Brasil. SBC.

Pastório, A., Rodrigues, L., and de Camargo, E. (2020). Uma revisão sistemática da literatura sobre tolerância a falhas em internet das coisas. In Anais Estendidos do X Sbesc, pages 57-64, Porto Alegre, RS, Brasil. SBC.

Rossato, J., Spanhol, F., and Camargo, E. (2020). Implantação e avaliação de uma rede sem-fio de longo alcance e baixa potência para cidades inteligentes. In Anais do IV Courb, pages 192-205, Porto Alegre, RS, Brasil. SBC. 